\section{Don't Give Up - They Eventually Grow Up: Issues in AYA Medicine}

\author{
Peter F. Coccia, MD
}

The sign in the Adolescent - Young Adult (AYA) diabetes clinic at The Nebraska Medical Center, reading "Don't Give Up - They Eventually Grow Up," reminded me that oncology professionals are not alone in dealing with the challenges and frustrations of caring for the AYA patient population.

For example, AYAs with type 1 diabetes have higher hemoglobin A1c levels than younger children. When cystic fibrosis patients become AYAs, they frequently demonstrate deteriorating pulmonary function with a significant decrement in FEV (forced expiratory volume). Also, solid organ transplant recipients have an increased risk of graft rejection when they become AYAs.

The NCI's AYA Oncology Progress Review Group, ASCO's Focus Under Forty program, the LIVESTRONG Young Adult Alliance, and the NCCN Clinical Practice Guidelines in Oncology (NCCN Guidelines) for AYA Oncology all define the AYA population as oncology patients who are 15 to 39 years of age at diagnosis. Although other medical specialties may use a lower upper age limit, an age approximating the onset of puberty defines the lower age limit.

There are certainly many host factors, including disease biology distinctions, genetic variants, developmental stage, pharmacologic considerations, and coexisting morbidities, that influence disease management and prognosis in AYA patients. However, the AYA population also has unique social, psychological, and economic issues that are shared across most disease conditions and that have major influences on morbidity and mortality. The NCCN Guidelines for AYA Oncology discuss in detail many of these issues, including the transition from family to independent living, the transition from pediatric to adult providers, financial difficulties (lack of employment, health insurance, transportation, and other resources), sexually transmitted diseases, gender identity, pregnancy, children, experimentation, and substance abuse. Discussion of these issues is beyond the scope of this commentary but are vitally important for AYA patients with chronic and life-threatening diseases.

However, the issues of compliance with and adherence to therapeutic recommendations by medical professionals are major problems across diseases and medical specialties and deserve elaboration. In oncology, nonadherence to selfadministered oral corticosteroids, mercaptopurine, and methotrexate in acute lymphoblastic leukemia and to imatinib in chronic myelogenous leukemia are commonly seen in AYA patients. Poor compliance with 1) subcutaneous insulin and dietary guidelines in type 1 diabetes, 2) the rigorous treatment regimens in cystic fibrosis, and 3) oral immunosuppression in solid organ transplant recipients may partially explain the previously mentioned examples of poor disease control. Other examples across specialties include poor adherence to oral antibiotic regimens; failure to comply with prolonged oral corticosteroid administration (with the frequent excuses being acne, weight gain, striae, and emotional lability); selling rather than taking medications for attention deficit hyperactivity disorder and sickle cell anemia; and frequent missed appointments for treatment or follow-up.

The transition from family-focused to patient-focused care, in my experience, is one of the major issues leading to decreased compliance. I think of it simplistically as the importance of "mothers" in assuring compliance: "Did you take your medicine?";

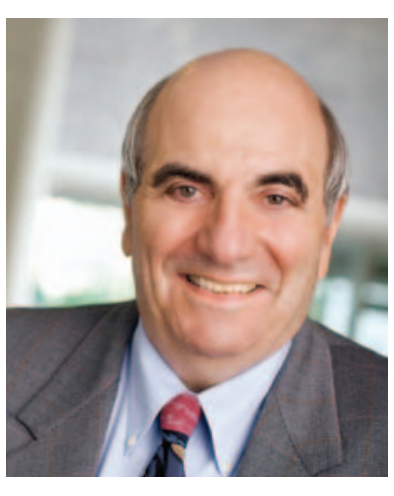

Peter F. Coccia, MD

Peter F. Coccia, MD, is the Ittner Professor and Vice-Chairman of the Department of Pediatrics, at the University of Nebraska Medical Center (UNMC) in Omaha.

Dr. Coccia received his medical degree from Upstate Medical Center where he also completed a predoctoral fellowship in biochemistry. His postgraduate training was in clinical pathology and hematopathology at Upstate and in pediatrics and pediatric hematology/oncology at the University of Minnesota. He served in the U.S. Public Health Service.

Dr. Coccia has been extensively involved in The Children's Cancer Group/Children's Oncology Group for more than 30 years as a PI and chairperson of numerous study committees and strategy groups. He is a member of the NCCN Board of Directors, and serves on the NCCN

Guidelines Panels for Adolescent and Young Adult Oncology (Chair), Acute Lymphoblastic Leukemia, and Cancer- and Chemotherapy-Induced Anemia. $\mathrm{He}$ is the author or coauthor of more than 170 peer-reviewed publications and more than 200 abstracts. His major clinical research areas have included the treatment of acute lymphoblastic leukemia and nonHodgkin's lymphomas in children and adolescents, and stem cell transplantation for inherited disorders, aplastic anemia, acute leukemia, and lymphomas.

The ideas and viewpoints expressed in this editorial are those of the author and do not necessarily represent any policy, position, or program of NCCN. 
"Here is your medicine, take it!"; "You have an appointment tomorrow, do you need a ride?"; "You feel warm; let's take your temperature."; "You have a fever; we are going to the emergency room." I further think that having a reliable support system (eg, mothers, spouses, significant others, friends) has a major influence on outcomes of therapy in many diseases. The absence of care partners argues strongly for the importance of multidisciplinary teams to assist in the management of AYA patients with serious illnesses. These teams may include nurses, nurse clinicians, physician assistants, patient navigators, social workers, psychologists, life specialists, financial counselors, clergy, and others, depending on the underlying condition and the patient's needs. And certainly the team members must be facile in all aspects of rapidly evolving social media networks.

The articles by Bleyer and by Zebrack and Butler in this issue of JNCCN expand on many of the subjects briefly mentioned in this commentary and also have applications to AYA diseases other than cancer. The NCCN Guidelines for AYA Oncology, also published in this issue, provide a framework for identifying issues and guidance for improving outcomes in AYA oncology patients. Representatives from all 21 NCCN Member Institutions served on the guidelines panel and included medical and pediatric oncologists, psychologists, and social workers with extensive interest and expertise in AYA oncology. The discussion section accompanying the guideline expands on the algorithms and includes detailed information concerning topics not covered in this commentary, including fertility/endocrine considerations, late effects of therapy, survivorship recommendations, palliative care and end-of-life considerations, and a listing of online resources for AYA patients. The reference list is comprehensive and current. As with all NCCN Guidelines, it will be revised yearly. Work is already underway on Version 1, 2013, which will certainly be improved and expanded. It will also benefit from extensive review at all 21 NCCN Member Institutions and will incorporate the suggestions of many additional experts. I am confident that the guidelines will be useful for practitioners in other medical specialties and provide insights for improving supportive care for AYA patients with many other diseases. 